\title{
A Symmetric Approach to the Massive Nonlinear Sigma Model
}

\author{
R. Ferrari ${ }^{a, 41}$ \\ ${ }^{a}$ Center for Theoretical Physics \\ Laboratory for Nuclear Science \\ and Department of Physics \\ Massachusetts Institute of Technology \\ Cambridge, Massachusetts 02139 and \\ ${ }^{b}$ Dip. di Fisica, Università degli Studi di Milano \\ and INFN, Sez. di Milano \\ via Celoria 16, I-20133 Milano, Italy \\ (MIT-CTP-4167, IFUM-961-FT, July, 2010)
}

\begin{abstract}
In the present paper we extend to the massive case the procedure of divergences subtraction, previously introduced for the massless nonlinear sigma model $(D=4)$. Perturbative expansion in the number of loops is successfully constructed. The resulting theory depends on the Spontaneous Symmetry Breaking parameter $v$, on the mass $m$ and on the radiative correction parameter $\Lambda$. Fermions are not considered in the present work. $S U(2) \otimes S U(2)$ is the group used.
\end{abstract}

\footnotetext{
${ }^{\dagger}$ This work is supported in part by funds provided by the U.S. Department of Energy (D.O.E.) under cooperative research agreement \#DE FG02-05ER41360

${ }^{1}$ e-mail: ruggero.ferrari@mi.infn.it
} 


\section{Introduction}

It is of paramount importance to establish a symmetric subtraction strategy for the divergences of the massive nonlinear sigma model. In fact this model provides a viable infrared regulator, a useful phenomenological theory and finally a template to approach more complex theories, as the nonabelian gauge theories in the 't Hooft gauge. Moreover a simulation on a lattice is free from the artifacts related to the presence of the zero modes.

The subtraction strategy for the ultraviolet divergences, devised for the massless nonlinear sigma model [1], can be successfully extended to the model with non zero mass, although chirality is broken.

A powerful Local Functional Equation (LFE) for the generating functionals (of the Green- and one-particle-irreducible-functions (1-PI)) ensues from the invariance properties of the path integral measure. By using the LFE, one can derive the complete hierarchy relations among the ancestor amplitudes (i.e. with no pion fields) and the descendant amplitudes (i.e. with at least one pion field) and get the full control of the divergences by a finite number of divergent amplitudes at each order in the loop expansion. The counterterms are chosen according to the symmetry properties of the effective action (not of the action!) fixed by the LFE. Pure pole subtraction in dimensional regularization turns out to be the right thing to do, in order to satisfy the LFE.

The physical parameters of the model are those of the classical action augmented by the scale of the radiative corrections $\Lambda$ (introduced later on).

To compare with the Chiral Perturbation Theory (ChPT) [2]-[5], we stress that the subtracted amplitudes depend on a fixed number of parameters. Moreover another important feature characterizes the present approach: $\Lambda$ is a physical parameter, while in ChPT the requirement of independence from $\Lambda$ leads to a Renormalization Group Equation.

\section{The Model}

The generating functionals $Z$ and $W$ are introduced via the path integral

$$
\begin{aligned}
& Z\left[\vec{K}, K_{0}, \vec{J}_{\mu}, N_{A B}\right]=\exp i W \simeq \int \mathcal{D}[\phi] \frac{1}{2 \phi_{0}} \exp [i S \\
& \left.+\Lambda^{D-4} \int d^{D} x\left(\phi_{0} K_{0}+\phi_{A} N_{A B} \phi_{B}\right)+\int d^{D} x \phi_{a} K_{a}\right] .
\end{aligned}
$$

The classical action is

$$
\begin{aligned}
& S=\Lambda^{D-4} \int d^{D} x \frac{v^{2}}{2}\left[\frac{1}{2} \operatorname{Tr}\left\{\left(F_{\mu}-J_{\mu}\right)^{2}\right\}-m^{2} \vec{\phi}^{2}\right] \\
& =\Lambda^{D-4} \int d^{D} x \frac{v^{2}}{2}\left[\frac{1}{4}\left(F_{a \mu}-J_{a \mu}\right)^{2}-m^{2} \vec{\phi}^{2}\right] \\
& =\Lambda^{D-4} \int d^{D} x \frac{v^{2}}{2}\left(\partial_{\mu} \phi_{A} \partial^{\mu} \phi_{A}-\frac{1}{2} F_{a \mu} J_{a}^{\mu}+\frac{1}{4} J^{2}-m^{2} \vec{\phi}^{2}\right) .
\end{aligned}
$$


where

$$
\begin{aligned}
& \phi_{0}=\sqrt{1-\vec{\phi}^{2}} \\
& \Omega=\phi_{A} \tau_{A}=\phi_{0}+i \phi_{a} \tau_{a}, \quad \Omega \in S U(2) \\
& F_{\mu}=\frac{\tau_{a}}{2} F_{a \mu} \equiv i \Omega \partial_{\mu} \Omega^{\dagger} \\
& F_{a \mu}=2\left(\phi_{0} \partial_{\mu} \phi_{a}-\partial_{\mu} \phi_{0} \phi_{a}+\epsilon_{a b c} \partial_{\mu} \phi_{b} \phi_{c}\right) \\
& \tau_{A} \equiv\{1, i \vec{\tau}\} .
\end{aligned}
$$

Capital letter indexes run over $\{0,1,2,3\}$ while lower cases over $\{1,2,3\}$. The fact that the fields $\phi_{0}, \vec{\phi}$ have null canonical dimension is only a matter of choice. $N_{A B}$ are real independent sources introduced in order to account for the extra composite operators generated by the local chiral transformations. By construction we have

$$
Z\left[\vec{K}, K_{0}, \vec{J}_{\mu}, N_{A B}\right]=Z\left[\vec{K}, K_{0}, \vec{J}_{\mu}, N_{B A}\right]
$$

i.e. $Z$ does not depend on the antisymmetric part of the matrix $N_{B A}$.

\section{$3 \quad$ The Local Functional Equation}

The measure in the path integral (1) is invariant under the local left-transformation

$$
\Omega^{\prime}=U(\vec{\omega}) \Omega
$$

i.e.

$$
\begin{aligned}
\delta \phi_{0} & =-\frac{\omega_{a}(x)}{2} \phi_{a} \\
\delta \phi_{a} & =\frac{\omega_{a}(x)}{2} \phi_{0}+\frac{\omega_{c}(x)}{2} \epsilon_{a b c} \phi_{b}
\end{aligned}
$$

for infinitesimal parameters $\vec{\omega}$.

The path integral is invariant under the field-coordinate transformation (6), thus we get an identity for the connected amplitude functional

$$
\begin{aligned}
& \int d^{D} x\left\langle\Lambda ^ { D - 4 } \left\{ v^{2}\left[\frac{1}{4}\left(F_{a \mu}-J_{a \mu}\right)\left(\epsilon_{a b c} F_{b}^{\mu} \omega_{c}+\partial^{\mu} \omega_{a}\right)-m^{2} \frac{\omega_{a}}{2} \phi_{a} \phi_{0}\right]\right.\right. \\
& -\frac{\omega_{a}}{2} \phi_{a} K_{0}-\frac{\omega_{a}}{2} \phi_{a} N_{0 B} \phi_{B}+\frac{\omega_{a}}{2} \phi_{0} N_{a B} \phi_{B}+\frac{\omega_{c}}{2} \epsilon_{a b c} \phi_{b} N_{a B} \phi_{B} \\
& \left.-\phi_{A} N_{A 0} \frac{\omega_{b}}{2} \phi_{b}+\phi_{A} N_{A a} \frac{\omega_{a}}{2} \phi_{0}+\phi_{A} N_{A b} \frac{\omega_{c}}{2} \epsilon_{b b^{\prime} c} \phi_{b^{\prime}}\right\} \\
& \left.+\frac{\omega_{a}}{2} \phi_{0} K_{a}+\frac{\omega_{c}}{2} \epsilon_{a b c} \phi_{b} K_{a}\right\rangle_{C}=0 .
\end{aligned}
$$


The brackets $\langle\cdot\rangle$ denote the mean value over the paths according to eq. (11). Then by using the symmetry (4)

$$
\begin{aligned}
& \left\{\left(-\epsilon_{a b c} J_{c}^{\mu}+\delta_{a b} \partial^{\mu}\right) \frac{\delta}{\delta J_{b}^{\mu}}-m^{2} \frac{v^{2}}{2} \frac{\delta}{\delta N_{a 0}}\right. \\
& -\frac{1}{2} N_{0 B} \frac{\delta}{\delta N_{a B}}+\frac{1}{2} N_{a B} \frac{\delta}{\delta N_{0 B}}-\frac{1}{2} \epsilon_{a b c} N_{c B} \frac{\delta}{\delta N_{b B}} \\
& -\frac{1}{2} N_{A 0} \frac{\delta}{\delta N_{A a}}+\frac{1}{2} N_{A a} \frac{\delta}{\delta N_{A 0}}-\frac{1}{2} \epsilon_{a b c} N_{A c} \frac{\delta}{\delta N_{A b}} \\
& \left.-\frac{\Lambda^{D-4}}{2} K_{0} \frac{\delta}{\delta K_{a}}+\frac{1}{2 \Lambda^{D-4}} K_{a} \frac{\delta}{\delta K_{0}}+\frac{1}{2} \epsilon_{a b c} K_{b} \frac{\delta}{\delta K_{c}}\right\} W=0 .
\end{aligned}
$$

We introduce the generators $L_{c A B}$ of the transformations (6)

$$
\begin{aligned}
& \delta \phi_{A}=\frac{1}{2} \omega_{c} L_{c A B} \phi_{B}, \quad L_{c 0 b}=-\delta_{c b}, L_{c a 0}=\delta_{c a}, L_{c a b}=\epsilon_{a b c} \\
& \omega_{c} L_{c}=\left(\begin{array}{llll}
0 & -\omega_{1} & -\omega_{2} & -\omega_{3} \\
\omega_{1} & 0 & \omega_{3} & -\omega_{2} \\
\omega_{2} & -\omega_{3} & 0 & \omega_{1} \\
\omega_{3} & \omega_{2} & -\omega_{1} & 0
\end{array}\right) \\
& {\left[L_{a}, L_{b}\right]=-2 \epsilon_{a b c} L_{c}}
\end{aligned}
$$

and we rewrite eq. (8) in the compact form

$$
\begin{aligned}
& \left\{\left(-\epsilon_{a b c} J_{c}^{\mu}+\delta_{a b} \partial^{\mu}\right) \frac{\delta}{\delta J_{b}^{\mu}}-m^{2} \frac{v^{2}}{2} \frac{\delta}{\delta N_{a 0}}\right. \\
& -\frac{1}{2}\left(L_{a A A^{\prime}} N_{A^{\prime} B}+L_{a B B^{\prime}} N_{A B^{\prime}}\right) \frac{\delta}{\delta N_{A B}} \\
& \left.-\frac{\Lambda^{D-4}}{2} K_{0} \frac{\delta}{\delta K_{a}}+\frac{1}{2 \Lambda^{D-4}} K_{a} \frac{\delta}{\delta K_{0}}+\frac{1}{2} \epsilon_{a b c} K_{b} \frac{\delta}{\delta K_{c}}\right\} W=0 .
\end{aligned}
$$

For the effective action (1-PI generating functional) one gets

$$
\begin{aligned}
& -\partial^{\mu} \Gamma_{J_{a}^{\mu}}-\epsilon_{a b c} J_{b}^{\mu} \Gamma_{J_{c}^{\mu}}+\frac{v^{2}}{2} m^{2} \Gamma_{N_{0 a}}+\frac{1}{2}\left(L_{a A A^{\prime}} N_{A^{\prime} B}+L_{a B B^{\prime}} N_{A B^{\prime}}\right) \Gamma_{N_{A B}} \\
& +\frac{\Lambda^{D-4}}{2} K_{0} \phi_{a}+\frac{1}{2 \Lambda^{D-4}} \Gamma_{K_{0}} \Gamma_{\phi_{a}}-\frac{1}{2} \epsilon_{a b c} \phi_{b} \Gamma_{\phi_{c}}=0,
\end{aligned}
$$

where we use the notation

$$
\Gamma_{X}=\frac{\delta}{\delta X} \Gamma
$$

\section{The Subtraction Strategy at $D=4$}

The LFE (11) is the tool we use in order to make finite the massive nonlinear sigma model. We work in the framework of the loop expansion of $\Gamma$ in eq. (11). 
This strategy has been employed with success for the massless nonlinear sigma model [6]-[10], for the massive Yang-Mills in the Landau gauge [11, [12, for a Higgsless Electroweak model also in the Landau gauge [13]-[15]. Therefore in models where dimensional regularization allows to drop the tadpoles involving the scalars fields $\vec{\phi}$. In the massive nonlinear sigma model the tadpoles play an important rôle and therefore it is necessary to extend the formalism to this case.

At the tree level $\Gamma^{(0)}$ is a solution of eq. (11) by construction. Dimensional regularization yields radiative corrections which do not generate any anomaly. The proof of this property is sketched in Ref. [1] and displayed in Ref. [9]. The Feynman rules are provided by the classical action $\Gamma^{(0)}$, while the counterterms $\hat{\Gamma}^{(k)}$ are introduced via the effective action which must obey the LFE (11). Therefore what matters are the symmetry properties of $\Gamma$ and not those of the action.

If the procedure of subtraction of infinities by means of the counterterms $\hat{\Gamma}^{(k)}$ has been carried out successfully up to order $n-1$, then

$$
\begin{aligned}
& -\partial^{\mu} \Gamma_{J_{a}^{\mu}}^{(k)}-\epsilon_{a b c} J_{b}^{\mu} \Gamma_{J_{c}^{\mu}}^{(k)}+\frac{v^{2}}{2} m^{2} \Gamma_{N_{0 a}}^{(k)}+\frac{1}{2}\left(L_{a A A^{\prime}} N_{A^{\prime} B}+L_{a B B^{\prime}} N_{A B^{\prime}}\right) \Gamma_{N_{A B}}^{(k)} \\
& +\frac{1}{2 \Lambda^{D-4}} \Gamma_{\phi_{a}}^{(0)} \Gamma_{K_{0}}^{(k)}+\frac{1}{2} \phi_{0} \Gamma_{\phi_{a}}^{(k)}-\frac{1}{2} \epsilon_{a b c} \phi_{b} \Gamma_{\phi_{c}}^{(k)}+\sum_{j=1}^{k-1} \frac{1}{2 \Lambda^{D-4}} \Gamma_{K_{0}}^{(k-j)} \Gamma_{\phi_{a}}^{(j)}=0, \forall k<n .
\end{aligned}
$$

At order $n$ we expect a violation of eq. (11)

$$
\begin{aligned}
& -\partial^{\mu} \Gamma_{J_{a}^{\mu}}^{(n)}-\epsilon_{a b c} J_{b}^{\mu} \Gamma_{J_{c}^{\mu}}^{(n)}+\frac{v^{2}}{2} m^{2} \Gamma_{N_{0 a}}^{(n)}+\frac{1}{2}\left(L_{a A A^{\prime}} N_{A^{\prime} B}+L_{a B B^{\prime}} N_{A B^{\prime}}\right) \Gamma_{N_{A B}}^{(k)} \\
& +\frac{1}{2 \Lambda^{D-4}} \Gamma_{\phi_{a}}^{(0)} \Gamma_{K_{0}}^{(n)}+\frac{1}{2} \phi_{0} \Gamma_{\phi_{a}}^{(n)}-\frac{1}{2} \epsilon_{a b c} \phi_{b} \Gamma_{\phi_{c}}^{(n)}+\sum_{j=1}^{n-1} \frac{1}{2 \Lambda^{D-4}} \Gamma_{K_{0}}^{(n-j)} \Gamma_{\phi_{a}}^{(j)} \\
& =\sum_{j=1}^{n-1} \frac{1}{2 \Lambda^{D-4}} \widehat{\Gamma}_{K_{0}}^{(n-j)} \widehat{\Gamma}_{\phi_{a}}^{(j)} .
\end{aligned}
$$

By assumption $\Gamma^{(k<n)}$ is finite, thus the removal of the poles in the Laurent expansion of

$$
\frac{1}{\Lambda^{D-4}} \Gamma^{(n)}
$$

is a strategy that maintains the validity of the LFE, since the RHS of eq. (14) is a pure pole part, when the normalization (15) is used. The finite part in the limit $D=4$ is the subtracted amplitude. It should be stressed that no further finite subtraction is allowed (even those keeping eq. (14) unchanged). In fact by adding extra counterterms one modifies the pure pole structure of the breaking terms. For instance on-shell renormalization is not a doable procedure. Finally the spotted counterterms $\widehat{\Gamma}^{(n)}$ obey the equation

$$
\begin{aligned}
& -\partial^{\mu} \widehat{\Gamma}_{J_{a}^{\mu}}^{(n)}-\epsilon_{a b c} J_{b}^{\mu} \widehat{\Gamma}_{J_{c}^{\mu}}^{(n)}+\frac{v^{2}}{2} m^{2} \widehat{\Gamma}_{N_{0 a}}^{(n)}+\frac{1}{2}\left(L_{a A A^{\prime}} N_{A^{\prime} B}+L_{a B B^{\prime}} N_{A B^{\prime}}\right) \widehat{\Gamma}_{N_{A B}}^{(k)} \\
& +\frac{1}{2 \Lambda^{D-4}} \widehat{\Gamma}_{\phi_{a}}^{(0)} \widehat{\Gamma}_{K_{0}}^{(n)}+\frac{1}{2} \phi_{0} \widehat{\Gamma}_{\phi_{a}}^{(n)}-\frac{1}{2} \epsilon_{a b c} \phi_{b} \widehat{\Gamma}_{\phi_{c}}^{(n)}+\sum_{j=1}^{n-1} \frac{1}{2 \Lambda^{D-4}} \widehat{\Gamma}_{K_{0}}^{(n-j)} \widehat{\Gamma}_{\phi_{a}}^{(j)}=0 .
\end{aligned}
$$


The last equation (16) provides a a posteriori explanation of why the breaking term takes the form exhibited in (14). A direct proof is provided in Ref. [9]. The subtraction strategy has been tested for a solvable model in Ref. [16.

\section{The Algebraic Aspects of the LFE}

It is convenient to define a new functional

$$
\mathfrak{G}\left[\phi_{a}, J_{a}^{\mu}, K_{0}, N_{00}, N_{0 a}, N_{a b}\right] \equiv \Gamma\left[\phi_{a}, J_{a}^{\mu}, K_{0}, N_{00}-\frac{1}{2} v^{2} m^{2}, N_{0 a}, N_{a b}\right]
$$

in presence of the nontrivial background

$$
\begin{aligned}
& \phi_{a}=J_{a}^{\mu}=K_{0}=N_{0 a}=N_{a b}=0 \\
& N_{00}=\frac{1}{2} m^{2} v^{2}
\end{aligned}
$$

and of the boundary conditions

$$
\begin{aligned}
& \left.\frac{\delta \mathfrak{G}^{(0)}}{\delta K_{0}}\right|_{\phi_{a}=J_{a}^{\mu}=K_{0}=N_{0 a}=N_{a b}=\left[N_{00}-\frac{1}{2} v^{2} m^{2}\right]=0}=\Lambda^{D-4} \\
& \left.\frac{\delta \mathfrak{G}^{(0)}}{\delta N_{00}}\right|_{\phi_{a}=J_{a}^{\mu}=K_{0}=N_{0 a}=N_{a b}=\left[N_{00}-\frac{1}{2} v^{2} m^{2}\right]=0}=\Lambda^{D-4} .
\end{aligned}
$$

By using $\mathfrak{G}$ we get rid of the mass term in eq. (11) and all the subsequent equations. For the counterterms $\widehat{\mathfrak{G}}^{(n)}$ eq. (16) gives

$$
\mathcal{S}_{a} \widehat{\mathfrak{G}}^{(n)}=-\sum_{j=1}^{n-1} \frac{1}{2 \Lambda^{D-4}} \widehat{\mathfrak{G}}_{K_{0}}^{(n-j)} \widehat{\mathfrak{G}}_{\phi_{a}}^{(j)},
$$

where

$$
\begin{aligned}
& \mathcal{S}_{a} \equiv-\partial^{\mu} \frac{\delta}{\delta J_{a}^{\mu}}-\epsilon_{a b c} J_{b}^{\mu} \frac{\delta}{\delta J_{c}^{\mu}}+\frac{1}{2}\left(L_{a A A^{\prime}} N_{A^{\prime} B}+L_{a B B^{\prime}} N_{A B^{\prime}}\right) \frac{\delta}{\delta N_{A B}} \\
& +\frac{1}{2 \Lambda^{D-4}} \mathfrak{G}_{\phi_{a}}^{(0)} \frac{\delta}{K_{0}}+\frac{1}{2} \phi_{0} \frac{\delta}{\phi_{a}}-\frac{1}{2} \epsilon_{a b c} \phi_{b} \frac{\delta}{\phi_{c}} .
\end{aligned}
$$

It is convenient to introduce the operators

$$
\begin{aligned}
\mathcal{S}_{a}^{J} & \equiv-\partial^{\mu} \frac{\delta}{\delta J_{a}^{\mu}}-\epsilon_{a b c} J_{b}^{\mu} \frac{\delta}{\delta J_{c}^{\mu}} \\
\mathcal{S}_{a}^{N} & \equiv \frac{1}{2}\left(L_{a A C} N_{C B}+L_{a B D} N_{A D}\right) \frac{\delta}{\delta N_{A B}} \\
\mathcal{S}_{a}^{\phi} & \equiv \frac{1}{2} \phi_{0} \frac{\delta}{\delta \phi_{a}}-\frac{1}{2} \epsilon_{a b c} \phi_{b} \frac{\delta}{\delta \phi_{c}} \\
\mathcal{S}_{a}^{\mathfrak{G}} & \equiv \frac{1}{2 \Lambda^{D-4}} \mathfrak{G}_{\phi_{a}}^{(0)} \frac{\delta}{\delta K_{0}} .
\end{aligned}
$$


It is straightforward to verify the following relations

$$
\begin{aligned}
& {\left[\mathcal{S}_{a}^{J}(x), \mathcal{S}_{a^{\prime}}^{J}(y)\right]=\epsilon_{a a^{\prime} c} \delta(x-y) \mathcal{S}_{c}^{J}(x)} \\
& {\left[\mathcal{S}_{a}^{N}(x), \mathcal{S}_{a^{\prime}}^{N}(y)\right]=\epsilon_{a a^{\prime} c} \delta(x-y) \mathcal{S}_{c}^{N}(x)} \\
& {\left[\mathcal{S}_{a}^{\phi}(x), \mathcal{S}_{a^{\prime}}^{\phi}(y)\right]=\epsilon_{a a^{\prime} c} \delta(x-y) \mathcal{S}_{c}^{\phi}(x)}
\end{aligned}
$$

and finally

$$
\left[\mathcal{S}_{a}(x), \mathcal{S}_{a^{\prime}}(y)\right]=\epsilon_{a a^{\prime} c} \delta(x-y) \mathcal{S}_{c}(x)
$$

\subsection{The Consistency Condition}

From eqs. (20) and (24) we get the consistency condition that has to be obeyed by the counterterms

$$
\begin{aligned}
& \Delta_{a}(x) \equiv-\sum_{j=1}^{n-1} \widehat{\mathfrak{G}}_{K_{0}(x)}^{(n-j)} \widehat{\mathfrak{G}}_{\phi_{a}(x)}^{(j)} \\
& \mathcal{S}_{a}(x) \Delta_{a^{\prime}}(y)-\mathcal{S}_{a^{\prime}}(y) \Delta_{a}(x)=\delta(x-y) \epsilon_{a a^{\prime} c} \Delta_{c}(x)
\end{aligned}
$$

\subsection{The Local Solutions: the bleaching Method}

The counterterms $\widehat{\mathfrak{G}}^{(n)}$ are given by linear combinations of local monomials constructed in terms of fields $\vec{\phi}$ and sources $K_{0}, J_{a}^{\mu}, N_{A B}$. The construction of the counterterms proceeds via the evaluation of the pole parts of the amplitudes as in eq. (15) and consequently the fixing of the coefficients of the general solution of eq. (20). For one-loop corrections one needs just the solution for the associated homogeneous equation.

This task is made easy if we replace the above mentioned variables by suitably chosen composite local invariants. The general procedure in Ref. [10] is here applied straightforwardly.

The local invariant solutions can be constructed with the following fields and sources

$$
\begin{aligned}
& F_{a}^{\mu}-J_{a}^{\mu} \\
& \left.D^{\mu}[F](F-J)^{\nu}\right|_{a b}=\left(\partial_{\mu} \delta_{a b}-\epsilon_{a b c} F_{c}^{\mu}\right)\left(F_{b}^{\nu}-J_{b}^{\nu}\right)=\partial_{\mu} \delta_{a b}-\epsilon_{a b c} J_{c}^{\mu}\left(F_{b}^{\nu}-J_{b}^{\nu}\right) \\
& \phi_{A} \equiv\left\{\phi_{0}, \phi_{a}\right\} \\
& K_{A} \equiv\left\{K_{0},-\frac{1}{\Lambda^{D-4}} \mathfrak{G}_{\phi_{a}}^{(0)}\right\}, \quad-\left.\frac{1}{\Lambda^{D-4}} \mathfrak{G}_{\phi_{a}}^{(0)}\right|_{\vec{\phi}=0}=-\frac{v^{2}}{2} \partial_{\mu} J_{a}^{\mu}-N_{0 a}-N_{a 0} \\
& N_{A B} .
\end{aligned}
$$

(Notice $\left.\left.D^{\mu}[F](F-J)^{\nu}\right|_{a b}-\left.D^{\mu}[J](F-J)^{\nu}\right|_{a b}=-\epsilon_{a b c}\left(F_{c}^{\mu}-J_{c}^{\mu}\right)\left(F_{b}^{\nu}-J_{b}^{\nu}\right)\right)$.

$K_{A}$ and $\phi_{A}$ transform in the same way. In fact

$$
\begin{aligned}
\mathcal{S}_{a}(x) \mathfrak{G}_{\phi_{b}(y)}^{(0)} & =\frac{1}{2} \delta(x-y)\left\{-\delta_{a b} \Lambda^{D-4} K_{0}(y)+\epsilon_{b c a} \mathfrak{G}_{c}^{(0)}(y)\right\} \\
\mathcal{S}_{a}(x) K_{0}(y) & =\delta(x-y) \frac{1}{2 \Lambda^{D-4}} \mathfrak{G}_{\phi_{a}(y)}^{(0)} .
\end{aligned}
$$


The bleached variables can be constructed according to the transformation properties of the fields in eq. (26). For instance

$$
\begin{aligned}
& \mathfrak{J}_{\mu} \equiv \Omega^{\dagger}\left(J_{\mu}-F_{\mu}\right) \Omega=\Omega^{\dagger} J_{\mu} \Omega+i \Omega^{\dagger} \partial_{\mu} \Omega \\
& \partial_{\mu} \mathfrak{J}_{\nu}=\Omega^{\dagger}\left(\partial_{\mu}+\Omega \partial_{\mu} \Omega^{\dagger}\right)\left(J_{\nu}-F_{\nu}\right) \Omega=\Omega^{\dagger} \mathcal{D}_{\mu}[F]\left(J_{\nu}-F_{\nu}\right) \Omega, \\
& \mathfrak{K}_{0} \equiv K_{A} \phi_{A}=K_{0} \phi_{0}-\frac{1}{\Lambda^{D-4}} \mathfrak{G}_{\phi_{a}}^{(0)} \phi_{a} \\
& \mathfrak{N}_{\alpha \beta, \rho \sigma}=\Omega_{\alpha \alpha^{\prime}}^{\dagger}\left(\tau_{A}\right)_{\alpha^{\prime} \rho}\left(\tau_{B}^{\dagger}\right)_{\beta \beta^{\prime}} \Omega_{\beta^{\prime} \sigma} N_{A B} .
\end{aligned}
$$

The bleaching yields $\mathcal{S}_{a}$-invariant local variables. Moreover the mapping is invertible.

The actual construction of the counterterms can profit of further properties of the LFE that limit the number of independent divergent amplitudes. The next Section deals with this feature.

\section{Hierarchy and Weak Power Counting}

Eq. (11) is nonlinear in $\Gamma$. This allows us to grade the 1PI functions in a hierarchical way according to the number of external $\phi$ - legs. In fact we have

$$
\begin{aligned}
& \Gamma_{\phi_{a}}=\frac{\Lambda^{D-4}}{\Gamma_{K_{0}}}\left\{\epsilon_{a b c} \phi_{b} \Gamma_{\phi_{c}}+2 \partial^{\mu} \Gamma_{J_{a}^{\mu}}+2 \epsilon_{a b c} J_{b}^{\mu} \Gamma_{J_{c}^{\mu}}\right. \\
& \left.-v^{2} m^{2} \Gamma_{N_{0 a}}-\Lambda^{D-4} K_{0} \phi_{a}-\left(L_{a A A^{\prime}} N_{A^{\prime} B}+L_{a B B^{\prime}} N_{A B^{\prime}}\right) \Gamma_{N_{A B}}\right\}
\end{aligned}
$$

The derivative of eq.(29) with respect to any ancestor variable $\left(J_{a}^{\mu}, K_{0}, N_{A B}\right)$ yields all the descendant amplitudes involving one $\phi-$ field. In a recursive way one obtains all the descendant amplitudes from the ancestor ones (hierarchy).

This result is very important because, at fixed order in the loop expansion, the number of independent divergent ancestor amplitudes is finite. In fact, by simple dimensional analysis, one can show that the superficial degree of divergence of a 1-PI graph $G$ for ancestor and descendant variables is bounded by

$$
\delta(G) \leq n_{L}(D-2)+2-N_{J}-2\left(N_{K}+N_{N}\right)
$$

where $n_{L}$ is the number of loops and $N_{J}, N_{K}, N_{N}$ are the numbers of insertions of the ancestor variables $J_{a}^{\mu}, K_{0}, N_{A B}$. Thus for fix $n_{L}$ the number of independent divergent ancestor amplitudes is finite. The bound in eq. (30) does not depend on the number of external $\phi$ - legs; therefore, if the ancestor amplitudes are divergent, an infinite number of descendant will also be divergent. The divergent parts of the descendant amplitudes will not be independent, due to the hierarchy property.

The bound (30) comes from the following two relations

$$
\delta(G)=n_{L} D-2 I+N_{J}+\sum_{k} k V_{k}
$$




$$
n_{L}=I-N_{J}-\left(N_{K}+N_{N}\right)-\sum_{k} V_{k}+1
$$

where $V_{k}$ is the number of vertexes with $k$ derivatives and $I$ the number of internal lines. The inequality in eq. (30) comes from the fact that for the unsubtracted theory $k \leq 2$.

The WPC criterion consists in building the classical action $\Gamma^{(0)}$ such that the bound (30) is obeyed. It is not necessary to introduce in $\Gamma^{(0)}$ all possible terms that are allowed by the WPC criterion. For instance it is not necessary to introduce a $\phi^{4}$ interaction in the model considered in the present paper. This approach is at variance with the algebraic renormalization procedure where the Power Counting theorem requires that all allowed couplings should enter with independent parameters.

The WPC theorem says that the bound (30) is stable under the subtraction procedure described in Section 4 . The proof goes as follows. The counterterm $\widehat{\Gamma}^{(k)}$, of order $k$ in the loop expansion, is a finite sum of local monomials built with $J_{a}^{\mu}, K_{0}, N_{A B}$ sources, spacetime derivatives and $N_{\phi} \phi$ - fields. Each monomial $\mathcal{M}^{(k)}\left(J_{a}^{\mu}, K_{0}, N_{A B}, N_{\phi}, n_{d}\right)$ entering in $\widehat{\Gamma}^{(k)}$ is constructed from graphs that obey eq. (30) and therefore it satisfies the condition

$$
k(D-2)+2-n_{J}-2\left(n_{K}+n_{N}\right)-n_{d} \geq 0,
$$

where $n_{d}$ is the number of derivatives and $n_{J}, n_{K}, n_{N}$ are the numbers of times the sources enter in the monomial. When one of this monomial is inserted in a graph, then the relations in eqs. (31) are modified by an extra vertex with $n_{d}$ derivatives. Moreover the numbers of external sources become $N_{J}-n_{J}, N_{K}-n_{K}, N_{N}-n_{N}$. The superficial degree of divergence becomes

$$
\begin{aligned}
& \delta(G) \leq\left(n_{L}-k\right)(D-2)+n_{d}-\left(N_{J}-n_{J}\right)-2\left[\left(N_{K}-n_{K}\right)+\left(N_{N}-n_{N}\right)\right] \\
& \leq n_{L}(D-2)+2-N_{J}-2\left(N_{K}+N_{N}\right)
\end{aligned}
$$

by using eq. (32) in the last step. Thus WPC is stable under subtraction of divergences.

\section{Hierarchical Relations and Feynman Rules}

In this Section we discuss some of the hierarchical relations for the two-point functions. This is an example. More relations and Feynman rules are given in Appendix A.

By successive differentiation of eq. (11) we get

$$
\begin{aligned}
& \partial^{\mu} \Gamma_{J_{a}^{\mu} J_{b}^{\nu}}-\frac{v^{2}}{2} m^{2} \Gamma_{N_{0 a} J_{b}^{\nu}}-\frac{1}{2 \Lambda^{D-4}} \Gamma_{K_{0}} \Gamma_{\phi_{a} J_{b}^{\nu}}=0 \\
& \partial^{\mu} \Gamma_{J_{a}^{\mu} N_{0 b}}-\frac{v^{2}}{2} m^{2} \Gamma_{N_{0 a} N_{0 b}}-\frac{1}{2} \Gamma_{N_{a b}}+\frac{1}{2} \delta_{a b} \Gamma_{N_{00}}+\frac{1}{2} \epsilon_{a b c} \Gamma_{N_{0 c}} \\
& -\frac{1}{2 \Lambda^{D-4}} \Gamma_{K_{0}} \Gamma_{\phi_{a} N_{0 b}}=0 \\
& \partial^{\mu} \Gamma_{J_{a}^{\mu} \phi_{b}}-\frac{v^{2}}{2} m^{2} \Gamma_{N_{0 a} \phi_{b}}-\frac{1}{2 \Lambda^{D-4}} \Gamma_{K_{0}} \Gamma_{\phi_{a} \phi_{b}}=0
\end{aligned}
$$


and finally $\left(\partial^{\mu} \Gamma_{K_{0}}=0\right)$

$$
\begin{gathered}
\Gamma_{\phi_{a} \phi_{b}}=\frac{2 \Lambda^{D-4}}{\Gamma_{K_{0}}}\left(\partial^{\mu} \Gamma_{J_{a}^{\mu} \phi_{b}}-\frac{v^{2}}{2} m^{2} \Gamma_{N_{0 a} \phi_{b}}\right)=\left(\frac{2 \Lambda^{D-4}}{\Gamma_{K_{0}}}\right)^{2}\left(\partial^{\mu}\left[-\partial^{\sigma} \Gamma_{J_{a}^{\mu} J_{b}^{\sigma}}-\frac{v^{2}}{2} m^{2} \Gamma_{J_{a}^{\mu} N_{0 b}}\right]\right. \\
\left.-\frac{v^{2}}{2} m^{2}\left[-\partial^{\mu} \Gamma_{N_{0 a} J_{b}^{\mu}}-\frac{v^{2}}{2} m^{2} \Gamma_{N_{0 a} N_{0 b}}-\frac{1}{2} \Gamma_{N_{a b}}+\frac{1}{2} \delta_{a b} \Gamma_{N_{00}}\right]\right)
\end{gathered}
$$

In the massless case all the terms present in eq. (36) are zero. For nonzero mass the expected contributions should come from the tadpole integral

$$
\begin{aligned}
& B_{0} \equiv \frac{1}{v^{2}} \int \frac{d^{D} q}{(2 \pi)^{D}} \frac{1}{\left(q^{2}-m^{2}\right)} ; \\
& \frac{1}{\Lambda^{D-4} v^{2}} \int \frac{d^{D} q}{(2 \pi)^{D}} \frac{1}{\left(q^{2}-m^{2}\right)}=-\frac{i}{v^{2}} \frac{1}{(4 \pi)^{2}} \Gamma\left(1-\frac{D}{2}\right) m^{2}\left(\frac{m}{\Lambda \sqrt{4 \pi}}\right)^{D-4} \\
& =i \frac{m^{2}}{v^{2}} \frac{1}{(4 \pi)^{2}}\left[-\frac{2}{D-4}+1-\gamma+\ln \left(\frac{m^{2}}{\Lambda^{2}(4 \pi)}\right)\right]+\mathcal{O}(D-4) .
\end{aligned}
$$

In fact one has

$$
\begin{aligned}
& \Gamma_{K_{0}}^{(1)}=-\frac{3}{2} i B_{0} \\
& \Gamma_{N_{00}}^{(1)}=2 \Gamma_{K_{0}}^{(1)}=-3 i B_{0} \\
& p^{\mu} \Gamma_{J_{a}^{\mu} J_{b}^{\nu}}^{(1)}=-i \frac{\delta_{a b}}{4} p^{\mu} \int \frac{d^{D} q}{(2 \pi)^{D}} \frac{(2 q+p)_{\mu}(2 q+p)_{\nu}}{\left[(p+q)^{2}-m^{2}\right]\left[q^{2}-m^{2}\right]}=-i \frac{v^{2} \delta_{a b}}{2} p_{\nu} B_{0} \\
& \Gamma_{N_{a b}}^{(1)}=i \delta_{a b} B_{0} \\
& \Gamma_{N_{0 a} N_{0 b}}^{(1)}=\Gamma_{J_{a}^{\mu} N_{0 b}}^{(1)}=\Gamma_{N_{0 b}}^{(1)}=0 \\
& \Gamma_{J_{a \mu}(p) \phi_{b}}^{(1)}=\frac{v^{2}}{4} B_{0} p^{\mu} \delta_{a b} \\
& \Gamma_{\phi_{a} N_{0 b}}^{(1)}=\delta_{a b} \frac{5}{3} \Gamma_{K_{0}}^{(1)}=-\frac{5}{2} i B_{0} \delta_{a b} \\
& \Gamma_{\phi_{a} \phi_{b}}^{(1)}=i \delta_{a b} v^{2}\left(p^{2}+m^{2}\right) B_{0} .
\end{aligned}
$$

\subsection{Two-point Feynman Rules}

In the zero loop approximation the boundary conditions (18) and eqs. (34) and (35) provide the Feynman rules

$$
\begin{aligned}
& \Gamma_{J_{a}^{\mu} \phi_{b}}^{(0)}=i p_{\mu} \delta_{a b} \frac{\Lambda^{D-4} v^{2}}{2} \\
& \Gamma_{N_{0 a} \phi_{b}}^{(0)}=\delta_{a b} \Lambda^{D-4} \\
& \Gamma_{\phi_{a} \phi_{b}}^{(0)}=\delta_{a b} \Lambda^{D-4} v^{2}\left(p^{2}-m^{2}\right) .
\end{aligned}
$$

\section{On the One-loop Counterterms}

The results of the previous sections allow to extract finite quantities from dimensional regularized amplitudes. Moreover local counterterms can be constructed so that one has 
a recursive process of subtraction. Perturbative unitarity is guaranteed by construction, under the form of cutting equation (see for instance Ref. [17]). Moreover the LFE (20) and the consistency condition (25) allows a order-by-order check of the counterterms.

It is of some interest to look for the local solutions of the LFE (20) and of its associated homogeneous equation. In fact, when the solutions are known, one can obtain all the counterterms for the descendant amplitudes counterterms. Further on we provide an example for the one-loop approximation. On one side this subject is very instructive, but on the other side, as it will be clear later on, the study of the local invariant solutions becomes very complex at higher order in the perturbative expansion.

We list some of the monomials that can be associated to one-loop divergent amplitudes. According to the eq. (30) they are expected to have dimension 4 or less, if we consider the subtraction procedure at $D=4$. Any monomial that does not contain the $\phi$-field (also implicitly as in $F_{a \mu}$ or in $K_{A}$ ) is a sterile term, since it cannot be the ancestor of any descendant amplitude. These sterile terms cannot be neglected: some of them take care of the counterterms associated to tadpoles. Here are few examples of dimension four constructed by using the bleached operators (i.e. invariant under the local transformations generated by $\left.\mathcal{S}_{a}(x)\right)$. Moreover global $S U(2)_{R}$ invariance is imposed. The antisymmetric part of $N_{A B}$ does not appear in the counterterms.

Let us list first the possible counterterms present in the massless case [6]

$$
\begin{aligned}
\mathcal{I}_{1} & =\int d^{D} x\left[D_{\mu}[F](J-F)_{\nu}\right]_{a}\left[D^{\mu}[F](J-F)^{\nu}\right]_{a}, \\
\mathcal{I}_{2} & =\int d^{D} x\left[D_{\mu}[F](J-F)^{\mu}\right]_{a}\left[D_{\nu}[F](J-F)^{\nu}\right]_{a}, \\
\mathcal{I}_{3} & =\int d^{D} x \epsilon_{a b c}\left[D_{\mu}[F](J-F)_{\nu}\right]_{a}\left(J_{b}^{\mu}-F_{b}^{\mu}\right)\left(J_{c}^{\nu}-F_{c}^{\nu}\right) \\
\mathcal{I}_{4}= & \int d^{D} x\left(K_{A} \phi_{A}\right)^{2} \\
& =\int d^{D} x\left(K_{0} \phi_{0}-\frac{\phi_{a}}{\Lambda^{D-4}} \mathfrak{G}_{\phi_{a}}^{(0)}\right)^{2} \\
\mathcal{I}_{5}= & \int d^{D} x\left(K_{A} \phi_{A}\right)\left(J_{c}^{\mu}-F_{c}^{\mu}\right)^{2} \\
& =\int d^{D} x\left(K_{0} \phi_{0}-\frac{\phi_{a}}{\Lambda^{D-4}} \mathfrak{G}_{\phi_{a}}^{(0)}\right)\left(J_{c}^{\mu}-F_{c}^{\mu}\right)^{2} \\
\mathcal{I}_{6}= & \int d^{D} x\left(J_{a}^{\mu}-F_{a}^{\mu}\right)^{2}\left(J_{b}^{\nu}-F_{b}^{\nu}\right)^{2} \\
\mathcal{I}_{7}= & \int d^{D} x\left(J_{a}^{\mu}-F_{a}^{\mu}\right)\left(J_{a}^{\nu}-F_{a}^{\nu}\right)\left(J_{b \mu}-F_{b \mu}\right)\left(J_{b \nu}-F_{b \nu}\right) .
\end{aligned}
$$

Notice the identity

$$
2\left(\mathcal{I}_{1}-\mathcal{I}_{2}\right)-4 \mathcal{I}_{3}+\left(\mathcal{I}_{6}-\mathcal{I}_{7}\right)=\int d^{D} x \mathcal{G}_{a \mu \nu}[\mathfrak{J}] \mathcal{G}_{a}^{\mu \nu}[\mathfrak{J}]=\int d^{D} x \mathcal{G}_{a \mu \nu}[J] \mathcal{G}_{a}^{\mu \nu}[J]
$$

where

$$
\mathcal{G}_{a \mu \nu}[J] \equiv \partial_{\mu} J_{a \nu}-\partial_{\nu} J_{a \mu}+\epsilon_{a b c} J_{b \mu} J_{c \nu}
$$


The right hand term in eq. (48) is sterile: no descendant terms are generated. The calculation [6] for the massless nonlinear sigma model gives

$$
\begin{aligned}
& \left.\widehat{\Gamma}^{(1)}\right|_{\text {MASSLESS }}=\frac{1}{D-4} \frac{\Lambda^{D-4}}{(4 \pi)^{2}}\left[-\frac{1}{12}\left(\mathcal{I}_{1}-\mathcal{I}_{2}-\mathcal{I}_{3}\right)+\frac{1}{48}\left(\mathcal{I}_{6}+2 \mathcal{I}_{7}\right)\right. \\
& \left.+\frac{3}{2} \frac{1}{v^{4}} \mathcal{I}_{4}+\frac{1}{2} \frac{1}{v^{2}} \mathcal{I}_{5}\right] .
\end{aligned}
$$

These counterterms are expected to be present in the same combination in the massive case, since no tadpoles contribute to the invariants $\mathcal{I}_{1}-\mathcal{I}_{7}$.

For later use we display the local invariant

$$
\begin{aligned}
& \left.K_{A} \phi_{A}\right|_{N_{00} \rightarrow N_{00}-\frac{m^{2} v^{2}}{2}}=\frac{K_{0}}{\phi_{0}}-\phi_{a}\left(-v^{2} \square \phi_{a}\right. \\
& \left.-v^{2} \int d^{D} x\left[\frac{J_{b}^{\mu}}{4} \frac{\delta F_{b \mu}}{\delta \phi_{a}}\right]+N_{a B} \phi_{B}+\phi_{B} N_{B a}-2 \phi_{a}\left(N_{00}+\frac{m^{2} v^{2}}{2}\right)\right)
\end{aligned}
$$

and its ancestor content

$$
\left.K_{A} \phi_{A}\right|_{\vec{\phi}=0}=K_{0} .
$$

The massive case requires the introduction of the sources $N_{A B}$ thus we get a numerous set of new invariants. At the one-loop level we have the local invariants candidates for $\widehat{\mathfrak{G}}^{(1)}$

$$
\begin{aligned}
& \mathcal{I}_{8}=\int d^{D} x N_{C C} \\
& \mathcal{I}_{9}=\int d^{D} x K_{A} \phi_{A} \\
& \mathcal{I}_{10}=\int d^{D} x\left(J_{c}^{\mu}-F_{c}^{\mu}\right)^{2} \\
& \mathcal{I}_{11}=\int d^{D} x \phi_{A} N_{A B} \phi_{B} \\
& \mathcal{I}_{12}=\int d^{D} x \phi_{A}\left(N_{A B}+N_{B A}\right)\left(N_{B C}+N_{C B}\right) \phi_{C} \\
& \mathcal{I}_{13}=\int d^{D} x \phi_{A} N_{A B} \phi_{B} \phi_{C} N_{C D} \phi_{D} \\
& \mathcal{I}_{14}=\int d^{D} x K_{C} \phi_{C} \phi_{A} N_{A B} \phi_{B} \\
& \mathcal{I}_{15}=\int d^{D} x K_{A}\left(N_{A B}+N_{B A}\right) \phi_{B}, \\
& \left.K_{A}\left(N_{A B}+N_{B A}\right)\right|_{\vec{\phi}=0}=2 K_{0} N_{00}-\left(\frac{v^{2}}{2} \partial_{\mu} J_{a}^{\mu}+N_{0 a}+N_{a 0}\right)\left(N_{0 a}+N_{a 0}\right) \\
& \mathcal{I}_{16}=\int d^{D} x \phi_{A} N_{A B} \phi_{B}\left(J_{c}^{\mu}-F_{c}^{\mu}\right)^{2} \\
& \mathcal{I}_{17}=\int d^{D} x K_{C} \phi_{C} N_{A A} \\
& \mathcal{I}_{18}=\int d^{D} x\left(N_{A B} N_{A B}+N_{A B} N_{B A}\right)
\end{aligned}
$$




$$
\begin{aligned}
& \mathcal{I}_{19}=\int d^{D} x N_{A A}\left(J_{c}^{\mu}-F_{c}^{\mu}\right)^{2} \\
& \mathcal{I}_{20}=\int d^{D} x\left(N_{A B}+N_{B A}\right) \operatorname{Tr}\left\{\Omega^{\dagger}\left(F^{\mu}-J^{\mu}\right) \Omega \tau_{A}^{\dagger}\left(F_{\mu}-J_{\mu}\right) \tau_{B}\right\} \\
& \mathcal{I}_{21}=\int d^{D} x K_{A} K_{A},\left.\quad K_{A} K_{A}\right|_{\vec{\phi}=0}=K_{0}^{2}+\left(\frac{v^{2}}{2} \partial_{\mu} J_{a}^{\mu}+N_{0 a}+N_{a 0}\right)^{2} .
\end{aligned}
$$

Let us find the ancestor variables content of the invariant $\mathcal{I}_{20}$ by using

$$
\left[\tau_{A}^{\dagger}, \frac{\tau_{b}}{2}\right]=\delta_{A x} \epsilon_{x b c} \tau_{c}, \quad\left(N_{A B}+N_{B A}\right) \tau_{A}^{\dagger} \tau_{B}=2 N_{A B}
$$

We have

$$
\begin{aligned}
& \left.\mathcal{I}_{20}\right|_{\vec{\phi}=0}=\left(N_{A B}+N_{B A}\right) \operatorname{Tr}\left\{J^{\mu} \tau_{A}^{\dagger} J_{\mu} \tau_{B}\right\} \\
& =\left(N_{A B}+N_{B A}\right)\left(\frac{1}{2} J_{a}^{\mu} J_{a \mu} \delta_{A B}+\delta_{A x} \epsilon_{x y z} J_{y \mu} \operatorname{Tr}\left\{J^{\mu} \tau_{z} \tau_{B}\right\}\right) \\
& =\left(N_{A B}+N_{B A}\right)\left(\frac{1}{2} J_{a}^{\mu} J_{a \mu} \delta_{A B}-\delta_{A x} \epsilon_{x y z} J_{y \mu} \epsilon_{y^{\prime} z x^{\prime}} \delta_{x^{\prime} B} J_{y^{\prime}}^{\mu}\right) \\
& =\left(N_{A B}+N_{B A}\right)\left(\frac{1}{2} J_{a}^{\mu} J_{a \mu} \delta_{A B}+\delta_{A x} J_{y \mu}\left(\delta_{x y^{\prime}} \delta_{y x^{\prime}}-\delta_{x x^{\prime}} \delta_{y y^{\prime}}\right) \delta_{x^{\prime} B} J_{y^{\prime}}^{\mu}\right) \\
& =N_{A A} J_{a}^{\mu} J_{a \mu}+2 N_{a b} J_{a \mu} J_{b}^{\mu}-2 N_{b b} J_{a}^{\mu} J_{a \mu} \\
& =\left(N_{00}-N_{b b}\right) J_{a}^{\mu} J_{a \mu}+2 N_{a b} J_{a \mu} J_{b}^{\mu} .
\end{aligned}
$$

In Appendix $\mathrm{B}$ we evaluate the coefficients of the invariants listed in eq. (53). Finally the tadpoles, originating from the mass term, necessitate the following counterterms at one-loop, that add to those for the massless nonlinear sigma model in eq. (50)

$$
\begin{aligned}
\left.\widehat{\Gamma}^{(1)}\right|_{\text {TADPOLES }}= & \frac{1}{D-4} \frac{\Lambda^{D-4}}{(4 \pi)^{2}}\left[-2 \frac{m^{2}}{v^{2}} \mathcal{I}_{8}+2 \frac{m^{2}}{v^{2}} \mathcal{I}_{11}-\frac{1}{v^{4}} \mathcal{I}_{12}+8 \frac{1}{v^{4}} \mathcal{I}_{13}\right. \\
& \left.+8 \frac{1}{v^{4}} \mathcal{I}_{14}+\frac{2}{v^{2}} \mathcal{I}_{16}-2 \frac{1}{v^{4}} \mathcal{I}_{17}+\frac{1}{v^{4}} \mathcal{I}_{18}-\frac{1}{2 v^{2}} \mathcal{I}_{19}-\frac{1}{2 v^{2}} \mathcal{I}_{20}\right] .
\end{aligned}
$$

From the expression in eqs. (50) and (56) one can get all the one-loop counterterms by taking the relevant functional derivatives. As an example one gets

$$
\begin{aligned}
& \widehat{\Gamma}_{\phi_{a} \phi_{b}}^{(1)}=\delta_{a b} \frac{1}{D-4} \frac{\Lambda^{D-4}}{(4 \pi)^{2}}\left[-2 m^{4}+2 m^{4}-8 m^{4}-8 m^{2}\left(p^{2}-m^{2}\right)\right. \\
& \left.+8 m^{2} p^{2}+2 m^{2}\left(p^{2}-m^{2}\right)-2 m^{2} p^{2}-2 m^{2} p^{2}\right] \\
& =\delta_{a b} \frac{1}{D-4} \frac{\Lambda^{D-4}}{(4 \pi)^{2}}\left[-2 m^{2}\left(p^{2}+m^{2}\right)\right] .
\end{aligned}
$$

which agrees with the direct calculation in eq. (45).

\section{Conclusions}

The subtraction strategy, recently developed for the nonlinear sigma model and for the nonabelian gauge theories, is implemented here for the massive nonlinear sigma model 
(without fermions in this work). In the present paper the technique has been applied to the simple case of $S U(2) \otimes S U(2)$. The extension to other groups of transformations is expected to be straightforward.

The main tool is the LFE for the effective action, derived from the invariance properties of the path integral measure. The presence of a mass term requires the introduction of more sources coupled to additional composite operators. However this fact brings only to a more complex algebra, without diminishing the power of the LFE. The hierarchy still works so that all 1PI-amplitudes with external field-parameters $\vec{\phi}$ can be derived from those with only composite operators. The hierarchy allows to organize, at every order of the loop expansion, the infinite set of divergent amplitudes, so that their divergent parts can be expressed in term of a finite number of divergent amplitudes. The subtraction algorithm exploits this powerful property and it is based on the dimensional regularization and on the subtraction of the sole pole parts (no finite adjustments are allowed). The WPC is shown to be stable under the subtraction procedure. The linearized LFE suggests the use of powerful local $S U(2)$ symmetry properties (gauge-type) in order to study the form of the counterterms. The bleaching technique is very useful since it maps all external source monomials into invariant quantities, hence very handy objects for the final aim: the construction of the counterterms.

The resulting perturbative expansion yields amplitudes that depend on the mass $m$, on the Spontaneous Symmetry Breaking parameter $v$ and on the scale of the radiative corrections $\Lambda$.

\section{Acknowledgments}

The author is pleased to thank the Center for Theoretical Physics at MIT, Massachusetts, where he had the possibility to work on this research.

\section{A More on Hierarchical Relations}

We provide more relations and Feynman rules for the three- and four-point amplitudes. The approach is outlined in Section 7.

\section{A.1 For the Three-point Functions}

We perform further derivatives of eq. (11) and we get the following relations among the ancestor amplitudes

$$
\begin{aligned}
& \partial^{\mu} \Gamma_{J_{a}^{\mu} J_{b}^{\nu} J_{c}^{\sigma}}+\epsilon_{a b c^{\prime}} \Gamma_{J_{c^{\prime}}^{\nu} J_{c}^{\sigma}}+\epsilon_{a c c^{\prime}} \Gamma_{J_{c^{\prime}}^{\sigma} J_{b}^{\nu}}-\frac{1}{2} m^{2} \Gamma_{N_{0 a} J_{b}^{\nu} J_{c}^{\sigma}} \\
& -\frac{1}{2 \Lambda^{D-4}} \Gamma_{K_{0}} \Gamma_{\phi_{a} J_{b}^{\nu} J_{c}^{\sigma}}=0 .
\end{aligned}
$$




$$
\begin{gathered}
\partial^{\mu} \Gamma_{J_{a}^{\mu} J_{b}^{\nu} N_{0 c}}+\epsilon_{a b c^{\prime}} \Gamma_{J_{c^{\prime}}^{\nu} N_{0 c}}-\frac{1}{2} m^{2} \Gamma_{N_{0 a} J_{b}^{\nu} N_{0 c}}-\frac{1}{2} \Gamma_{N_{c a} J_{b}^{\nu}}+\frac{1}{2} \delta_{a c} \Gamma_{N_{00} J_{b}^{\nu}} \\
+\frac{1}{2} \epsilon_{a c c^{\prime}} \Gamma_{N_{0 c^{\prime}} J_{b}^{\nu}}-\frac{1}{2 \Lambda^{D-4} v^{2}} \Gamma_{K_{0}} \Gamma_{\phi_{a} J_{b}^{\nu} N_{0 c}}=0 . \\
\partial^{\mu} \Gamma_{J_{a}^{\mu} N_{0 b} N_{0 c}}-\frac{1}{2} m^{2} \Gamma_{N_{0 a} N_{0 b} N_{0 c}}-\frac{1}{2} \Gamma_{N_{b a} N_{0 c}}-\frac{1}{2} \Gamma_{N_{c a} N_{0 b}}+\frac{1}{2} \delta_{a b} \Gamma_{N_{00} N_{0 c}}+\frac{1}{2} \delta_{a c} \Gamma_{N_{00} N_{0 b}} \\
+\frac{1}{2} \epsilon_{a b c^{\prime}} \Gamma_{N_{0 c^{\prime}} N_{0 c}}+\frac{1}{2} \epsilon_{a c c^{\prime}} \Gamma_{N_{B c^{\prime}} N_{0 b}}-\frac{1}{2 \Lambda^{D-4} v^{2}} \Gamma_{K_{0}} \Gamma_{\phi_{a} N_{0 b} N_{0 c}}=0 .
\end{gathered}
$$

If one $\phi$ - derivative is taken, one has

$$
\begin{gathered}
\partial^{\mu} \Gamma_{J_{a}^{\mu} J_{b}^{\nu} \phi_{c}}+\epsilon_{a b c^{\prime}} \Gamma_{J_{c^{\prime}}^{\nu} \phi_{c}}-\frac{1}{2} m^{2} \Gamma_{N_{0 a} J_{b}^{\nu} \phi_{c}} \\
-\frac{1}{2 \Lambda^{D-4} v^{2}} \Gamma_{K_{0}} \Gamma_{\phi_{a} J_{b}^{\nu} \phi_{c}}+\frac{1}{2} \epsilon_{a c c^{\prime}} \Gamma_{\phi_{c^{\prime}} J_{b}^{\nu}}=0 . \\
\partial^{\mu} \Gamma_{J_{a}^{\mu} N_{0 b} \phi_{c}}-\frac{1}{2} m^{2} \Gamma_{N_{0 a} N_{0 b} \phi_{c}}-\frac{1}{2} \Gamma_{N_{b a} \phi_{c}} \\
+\frac{1}{2} \epsilon_{a b c^{\prime}} \Gamma_{N_{0 c^{\prime}} \phi_{c}}-\frac{1}{2 \Lambda^{D-4} v^{2}} \Gamma_{K_{0}} \Gamma_{\phi_{a} N_{0 b} \phi_{c}}+\frac{1}{2} \epsilon_{a c c^{\prime}} \Gamma_{\phi_{c^{\prime}} N_{0 b}}=0 . \\
\partial^{\mu} \Gamma_{J_{a_{1}}^{\mu} N_{a_{2} a_{3}} \phi_{a_{4}}}-\frac{1}{2} m^{2} \Gamma_{N_{0 a_{1}} N_{a_{2} a_{3}} \phi_{a_{4}}}+\frac{1}{2} \delta_{a_{1} a_{2}} \Gamma_{N_{0 a_{3}} \phi_{a_{4}}}+\frac{1}{2} \delta_{a_{1} a_{3}} \Gamma_{N_{a_{2} 0} \phi_{a_{4}}} \\
-\frac{1}{2 \Lambda^{D-4} v^{2}} \Gamma_{K_{0}} \Gamma_{\phi_{a_{1}} N_{a_{2} a_{3} \phi_{a_{4}}}+\frac{1}{2} \epsilon_{a_{1} a_{4} c^{\prime}} \Gamma_{\phi_{c^{\prime}} N_{a_{2} a_{3}}}=0 .}
\end{gathered}
$$

Two $\phi$ - derivatives yields

$$
\begin{aligned}
& \partial^{\mu} \Gamma_{J_{a}^{\mu} \phi_{b} \phi_{c}}-\frac{1}{2} m^{2} \Gamma_{N_{0 a} \phi_{b} \phi_{c}}-\frac{1}{2 \Lambda^{D-4} v^{2}}\left(\Gamma_{K_{0}} \Gamma_{\phi_{a} \phi_{b} \phi_{c}}+\Gamma_{K_{0} \phi_{b}} \Gamma_{\phi_{a} \phi_{c}}\right. \\
& \left.+\Gamma_{K_{0} \phi_{c}} \Gamma_{\phi_{a} \phi_{b}}+\Gamma_{K_{0} \phi_{c} \phi_{b}} \Gamma_{\phi_{a}}\right)+\frac{1}{2} \epsilon_{a b c^{\prime}} \Gamma_{\phi_{c^{\prime}} \phi_{c}}+\frac{1}{2} \epsilon_{a c c^{\prime}} \Gamma_{\phi_{c^{\prime}} \phi_{b}}=0 .
\end{aligned}
$$

Thus one can obtain all the amplitudes involving the $\phi$ - fields $\left(\Gamma_{\phi_{a} \phi_{b} \phi_{c}}=0\right)$.

\section{A.2 Three-point Feynman Rules}

From eq. (11) we get

$$
\Gamma_{K_{0} \phi_{a} \phi_{b}}^{(0)}=-\delta_{a b} \Lambda^{D-4}
$$

From eqs. (61) and (62) we get

$$
\begin{aligned}
& \Gamma_{J_{a}^{\mu} \phi_{b} \phi_{c}}^{(0)}=-\frac{i}{2} \Lambda^{D-4} v^{2} \epsilon_{a b c}\left(p_{b}-p_{c}\right)_{\mu} \\
& \Gamma_{N_{0 a} \phi_{b} \phi_{c}}^{(0)}=0 \\
& \Gamma_{\phi_{a_{1}} N_{a_{2} a_{3} \phi_{a_{4}}}}^{(0)}=\Lambda^{D-4}\left(\delta_{a_{1} a_{2}} \delta_{a_{4} a_{3}}+\delta_{a_{1} a_{3}} \delta_{a_{4} a_{2}}\right)
\end{aligned}
$$




\section{A.3 For the four-point Functions}

We consider also the four-point functions, but we derive only the relations that are necessary in order to get the Feynman rules used in the present paper. We get the following identities from eq. (11)

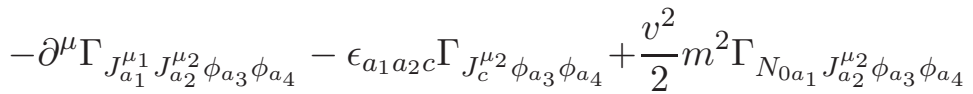

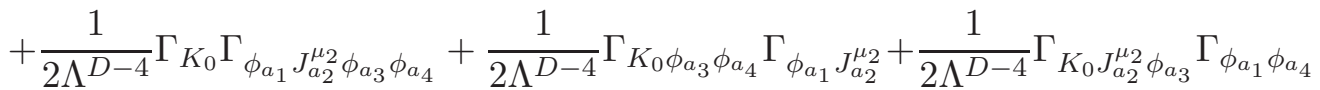

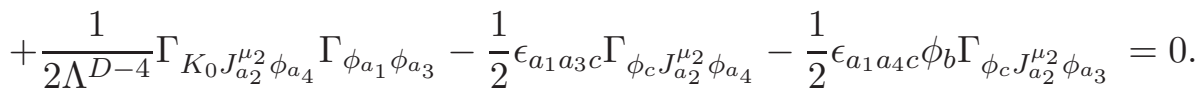

We need also

$$
\begin{aligned}
& -\partial^{\mu} \Gamma_{J_{a_{1}}^{\mu_{1}} N_{0 a_{2}} \phi_{a_{3} \phi_{a_{4}}}}+\frac{v^{2}}{2} m^{2} \Gamma_{N_{0 a_{1}} N_{0 a_{2}} \phi_{a_{3}} \phi_{a_{4}}}+\frac{1}{2} \Gamma_{N_{a_{1} a_{2}} \phi_{a_{3}} \phi_{a_{4}}}-\frac{1}{2} \delta_{a_{1} a_{2}} \Gamma_{N_{00} \phi_{a_{3}} \phi_{a_{4}}} \\
& +\frac{1}{2 \Lambda^{D-4}} \Gamma_{K_{0}} \Gamma_{\phi_{a_{1}} N_{0 a_{2}} \phi_{a_{3}} \phi_{a_{4}}}+\frac{1}{2 \Lambda^{D-4}} \Gamma_{K_{0} \phi_{a_{3}} \phi_{a_{4}}} \Gamma_{\phi_{a_{1}} N_{0 a_{2}}} \\
& -\frac{1}{2} \epsilon_{a_{1} a_{3} c} \Gamma_{\phi_{c} N_{0 a_{2}} \phi_{a_{4}}}-\frac{1}{2} \epsilon_{a_{1} a_{4} c} \phi_{b} \Gamma_{\phi_{c} N_{0 a_{2}} \phi_{a_{3}}}=0
\end{aligned}
$$

Thus finally we can obtain $\Gamma_{\phi_{a_{1}} \phi_{a_{2}} \phi_{a_{3}} \phi_{a_{4}}}$ from

$$
\begin{aligned}
& -\partial^{\mu} \Gamma_{J_{a_{1}}^{\mu_{1}} \phi_{a_{2}} \phi_{a_{3}} \phi_{a_{4}}}+\frac{v^{2}}{2} m^{2} \Gamma_{N_{0 a_{1}} \phi_{a_{2}} \phi_{a_{3}} \phi_{a_{4}}}+\frac{1}{2 \Lambda^{D-4}} \Gamma_{K_{0}} \Gamma_{\phi_{a_{1}} \phi_{a_{2}} \phi_{a_{3}} \phi_{a_{4}}} \\
& +\frac{1}{2 \Lambda^{D-4}} \sum_{j=2}^{4} \Gamma_{K_{0} \phi_{a_{j+1}} \phi_{a_{j+2}}} \Gamma_{\phi_{a_{1}} \phi_{a_{j}}}=0 .
\end{aligned}
$$

\section{A.4 Four-point Feynman Rules}

From the above relations (67 [69) at zero loop we get the Feynman rules

$$
\begin{aligned}
& \Gamma_{\phi_{a_{1}} J_{a_{2}}^{\mu_{2}} \phi_{a_{3} \phi_{a_{4}}}}^{(0)}= \\
& i \frac{\Lambda^{D-4} v^{2}}{2}\left(\delta_{a_{1} a_{2}} \delta_{a_{3} a_{4}}\left(2 p_{1}+p_{2}\right)+\delta_{a_{1} a_{3}} \delta_{a_{4} a_{2}}\left(p_{2}+2 p_{4}\right)+\delta_{a_{1} a_{4}} \delta_{a_{2} a_{3}}\left(2 p_{3}+p_{2}\right)\right)_{\mu_{2}}
\end{aligned}
$$

and

$$
\Gamma_{\phi_{a_{1}} N_{0 a_{2}} \phi_{a_{3}} \phi_{a_{4}}}^{(0)}=-\Lambda^{D-4}\left(\delta_{a_{1} a_{2}} \delta_{a_{3} a_{4}}+\delta_{a_{1} a_{3}} \delta_{a_{4} a_{2}}+\delta_{a_{1} a_{4}} \delta_{a_{2} a_{3}}\right)
$$

Finally from eqs. (69), (70) and (71)

$$
\begin{aligned}
& \Gamma_{\phi_{a_{1}} \phi_{a_{2}} \phi_{a_{3} \phi_{a_{4}}}}^{(0)}= \\
& \Lambda^{D-4} v^{2}\left(\delta_{a_{1} a_{2}} \delta_{a_{3} a_{4}}\left(p_{2}+p_{1}\right)^{2}+\delta_{a_{2} a_{3}} \delta_{a_{4} a_{1}}\left(p_{1}+p_{4}\right)^{2}+\delta_{a_{2} a_{4}} \delta_{a_{1} a_{3}}\left(p_{3}+p_{1}\right)^{2}\right) .
\end{aligned}
$$

In this Appendix we have verified that the Feynman rules are those given by the zero loop effective action $\Gamma^{(0)}$. 


\section{B Evaluation of the Counterterms}

In this Appendix we evaluate the coefficients of the invariants listed in eq. (53) by comparing their external sources content (i.e. ancestor amplitudes) with the one-loop calculations given in eqs. (38,42) and with the coefficients in eq. (50).

\section{B.1 Ancestor Invariants}

It should be reminded that the counterterms in eqs. (50) and (53) are used as extra Feynman rules after the final substitution $N_{00} \rightarrow N_{00}+\frac{1}{2} m^{2} v^{2}$.

- $\underline{N-J}$ amplitudes. Since at the one-loop level there is no $\widehat{\Gamma}_{J_{b}^{\mu} N_{0 a}}^{(1)}$, we have

$$
a_{15}=2 a_{21}
$$

in fact, as shown in eq. (26), $K_{A}$ contains both $N_{0 a}$ and $J_{b}^{\mu}$. This term can be neglected since it can be written in terms of other invariants

$$
\left.\left(2 \mathcal{I}_{15}+\mathcal{I}_{21}\right)\right|_{\vec{\phi}=0}=\left.\left(\frac{v^{4}}{4} \mathcal{I}_{2}+\mathcal{I}_{4}-\mathcal{I}_{12}+4 \mathcal{I}_{14}\right)\right|_{\vec{\phi}=0}
$$

- $N-J-J$ amplitudes. We consider the generic combination

$$
a_{10} \mathcal{I}_{10}+a_{16} \mathcal{I}_{16}+a_{19} \mathcal{I}_{19}+a_{20} \mathcal{I}_{20}
$$

At one-loop level we have

$$
\begin{aligned}
& \widehat{\Gamma}_{N_{00} J_{c}^{\mu} J_{d}^{\nu}}^{(1)}=2 \widehat{\Gamma}_{K_{0} J_{c}^{\mu} J_{d}^{\nu}}^{(1)}=\frac{2}{v^{2}} g_{\mu \nu} \delta_{c d} \\
& \widehat{\Gamma}_{N_{a b} J_{c}^{\mu} J_{d}^{\nu}}^{(1)}=-\left(\delta_{a c} \delta_{b d}+\delta_{a d} \delta_{b c}\right) \frac{1}{v^{2}} g_{\mu \nu} .
\end{aligned}
$$

Thus we get

$$
\begin{aligned}
& a_{16}+a_{19}+a_{20}=\frac{1}{v^{2}} \\
& a_{19}=a_{20} \\
& a_{20}=-\frac{1}{2 v^{2}} \\
& a_{16}=\frac{2}{v^{2}} .
\end{aligned}
$$

- $J-J$ amplitudes. Now we can check the content of eq. (75)

$$
a_{10} \mathcal{I}_{10}+\frac{1}{2 v^{2}}\left(4 \mathcal{I}_{16}-\mathcal{I}_{19}-\mathcal{I}_{20}\right)
$$

to be compared with the expected counterterm coming from eq. (40), i.e. $m^{2}$. We get

$$
2 a_{10}+\frac{m^{2} v^{2}}{2} \frac{1}{2 v^{2}} 2(4-1-1)=m^{2}
$$


Thus the final combination is

$$
\frac{1}{2 v^{2}}\left(4 \mathcal{I}_{16}-\mathcal{I}_{19}-\mathcal{I}_{20}\right)
$$

- $N-N$ amplitudes. Now we consider the invariants that contain $N_{A B}$. The $N_{00}$ is coupled to $\vec{\phi}^{2}$, while $N_{0 a}$ to $\vec{\phi}^{2} \phi_{a}$ and finally $N_{a b}$ to $\phi_{a} \phi_{b}$. Thus there is no countertems for $\Gamma_{N_{00} N_{0 a}}$ and $\Gamma_{N_{a a} N_{0 b}}$. Finally at one loop there no divergence for $\Gamma_{N_{0 b} N_{0 a}}^{(1)}$. We group together all invariants with ancestor amplitude containing only $N_{A B}$

$$
a_{8} \mathcal{I}_{8}+a_{11} \mathcal{I}_{11}+a_{12} \mathcal{I}_{12}+a_{13} \mathcal{I}_{13}+a_{18} \mathcal{I}_{18}
$$

The absence of bilinear terms involving $N_{0 a}$ requires the combination

$$
a_{8} \mathcal{I}_{8}+a_{11} \mathcal{I}_{11}+a_{12}\left(\mathcal{I}_{12}-\mathcal{I}_{18}\right)+a_{13} \mathcal{I}_{13}
$$

In fact

$$
\begin{aligned}
& \left.\left(\mathcal{I}_{12}-\mathcal{I}_{18}\right)\right|_{\vec{\phi}=0}=\left(2 \phi_{A} N_{A B} N_{B C} \phi_{C}-N_{A B} N_{B A}\right. \\
& \left.+\phi_{A} N_{A B} N_{C B} \phi_{C}+\phi_{A} N_{B A} N_{B C} \phi_{C}-N_{A B} N_{A B}\right)\left.\right|_{\vec{\phi}=0} \\
& =2 N_{00}^{2}+2 N_{0 a} N_{a 0}-N_{00}^{2}-2 N_{0 a} N_{a 0}-N_{a b} N_{b a}+N_{00}^{2}+N_{0 a} N_{0 a} \\
& +N_{00}^{2}+N_{a 0} N_{a 0}-N_{00}^{2}-N_{0 a} N_{0 a}-N_{a 0} N_{a 0}-N_{a b} N_{a b} \\
& =2 N_{00}^{2}-\left(N_{a b} N_{a b}+N_{a b} N_{b a}\right)
\end{aligned}
$$

Thus we get

$$
\begin{aligned}
& \widehat{\Gamma}_{N_{00} N_{00}}^{(1)}=4 a_{12}+2 a_{13}=4 \widehat{\Gamma}_{K_{0} K_{0}}^{(1)}=\frac{12}{v^{4}} \\
& \widehat{\Gamma}_{N_{a a^{\prime}} N_{b b^{\prime}}}^{(1)}=-2 a_{12}\left(\delta_{a b} \delta_{a^{\prime} b^{\prime}}+\delta_{a b^{\prime}} \delta_{b a^{\prime}}\right)=\left(\delta_{a b} \delta_{a^{\prime} b^{\prime}}+\delta_{a b^{\prime}} \delta_{b a^{\prime}}\right) \frac{2}{v^{4}}
\end{aligned}
$$

i.e.

$$
a_{12}=-\frac{1}{v^{4}}, \quad a_{13}=\frac{8}{v^{4}}
$$

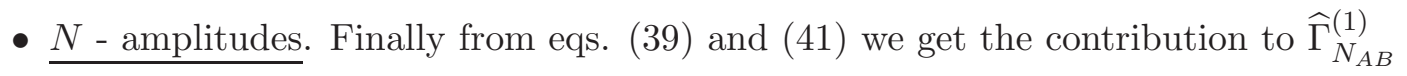

$$
\begin{aligned}
& a_{8}+a_{11}+m^{2} v^{2}\left(2 a_{12}+a_{13}\right)=6 \frac{m^{2}}{v^{2}} \\
& a_{8} \delta_{a b}=-2 \frac{m^{2}}{v^{2}} \delta_{a b}, \quad a_{11}=2 \frac{m^{2}}{v^{2}} .
\end{aligned}
$$

Finally

$$
-2 \frac{m^{2}}{v^{2}} \mathcal{I}_{8}+2 \frac{m^{2}}{v^{2}} \mathcal{I}_{11}-\frac{1}{v^{4}}\left(\mathcal{I}_{12}-\mathcal{I}_{18}\right)+\frac{8}{v^{4}} \mathcal{I}_{13}
$$


- $K_{0}-N$ amplitudes. We consider the linear combination

$$
a_{9} \mathcal{I}_{9}+a_{14} \mathcal{I}_{14}+a_{17} \mathcal{I}_{17}
$$

We impose the conditions using eq. (50)

$$
\begin{aligned}
& \widehat{\Gamma}_{K_{0} N_{00}}^{(1)}=a_{14}+a_{17}=2 \widehat{\Gamma}_{K_{0} K_{0}}^{(1)}=\frac{6}{v^{4}} \\
& \widehat{\Gamma}_{K_{0} N_{a b}}^{(1)}=a_{17} \delta_{a b}=-2 \frac{1}{v^{4}} \delta_{a b} .
\end{aligned}
$$

We get

$$
a_{17}=-\frac{2}{v^{4}}, \quad a_{14}=\frac{8}{v^{4}} .
$$

The tadpole term in eq. (38) requires

$$
a_{9}+\frac{m^{2} v^{2}}{2} a_{14}+\frac{m^{2} v^{2}}{2} a_{17}=\frac{3 m^{2}}{v^{2}}, \quad \Longrightarrow a_{9}=0 .
$$

Finally we have

$$
\frac{8}{v^{4}} \mathcal{I}_{14}-\frac{2}{v^{4}} \mathcal{I}_{17}
$$

The result of the Appendix is summarized in eq. (56).

\section{References}

[1] R. Ferrari, JHEP 0508, 048 (2005) arXiv:hep-th/0504023.

[2] S. Weinberg, Physica A 96 (1979) 327.

[3] G. Colangelo, Phys. Lett. B 350, 85 (1995) [Erratum-ibid. B 361, 234 (1995)] arXiv:hep-ph/9502285.

[4] J. Bijnens, G. Colangelo and G. Ecker, Phys. Lett. B 441, 437 (1998) arXiv:hep-ph/9808421.

[5] J. Bijnens and L. Carloni, Nucl. Phys. B 827, 237 (2010) [arXiv:0909.5086 [hep-ph]].

[6] R. Ferrari and A. Quadri, Int. J. Theor. Phys. 45, 2497 (2006) arXiv:hep-th/0506220.

[7] R. Ferrari and A. Quadri, JHEP 0601, 003 (2006) arXiv:hep-th/0511032.

[8] D. Bettinelli, R. Ferrari and A. Quadri, Int. J. Theor. Phys. 46 (2007) 2560 arXiv:hep-th/0611063.

[9] D. Bettinelli, R. Ferrari and A. Quadri, Int. J. Mod. Phys. A 23, 211 (2008) arXiv:hep-th/0701197.

[10] D. Bettinelli, R. Ferrari and A. Quadri, JHEP 0703, 065 (2007) arXiv:hep-th/0701212. 
[11] D. Bettinelli, R. Ferrari and A. Quadri, Phys. Rev. D 77, 045021 (2008) arXiv:0705.2339 [hep-th]].

[12] D. Bettinelli, R. Ferrari and A. Quadri, Phys. Rev. D 77 (2008) 105012 arXiv:0709.0644 [hep-th]].

[13] D. Bettinelli, R. Ferrari and A. Quadri, Int. J. Mod. Phys. A 24 (2009) 2639 arXiv:0807.3882 [hep-ph]].

[14] D. Bettinelli, R. Ferrari and A. Quadri, Acta Phys. Pol. B 41, 597 (2010) arXiv:0809.1994 [hep-th]].

[15] D. Bettinelli, R. Ferrari and A. Quadri, Phys. Rev. D 79, 125028 (2009) arXiv:0903.0281 [hep-th]].

[16] R. Ferrari, J. Math. Phys. 51, 032305 (2010) arXiv:0907.0426 [hep-th]].

[17] G. 't Hooft and M. J. G. Veltman, NATO Adv. Study Inst. Ser. B Phys. 4 (1974) 177. 